Correspondence Francisco García-del Portillo fgportillo@cnb.csic.es
Received 12 March 2013 Accepted 2 May 2013

\section{Occurrence of mutations impairing sigma factor B (SigB) function upon inactivation of Listeria monocytogenes genes encoding surface proteins}

\author{
Juan J. Quereda, ${ }^{1}$ M. Graciela Pucciarelli, ${ }^{1,2}$ Laura Botello-Morte, ${ }^{1}$ \\ Enrique Calvo, ${ }^{3}$ Filipe Carvalho, ${ }^{4}$ Christiane Bouchier, ${ }^{5}$ Ana Vieira, ${ }^{4}$ \\ Javier F. Mariscotti, ${ }^{1}$ Trinad Chakraborty, ${ }^{6}$ Pascale Cossart, ${ }^{7}$ \\ Torsten Hain, ${ }^{6}$ Didier Cabanes ${ }^{4}$ and Francisco García-del Portillo ${ }^{1}$
}

1Centro Nacional de Biotecnología-Consejo Superior de Investigaciones Científicas (CNB-CSIC),
28049 Madrid, Spain
${ }^{2}$ Departamento de Biología Molecular, Universidad Autónoma de Madrid. Centro de Biología
Molecular 'Severo Ochoa' (CBMSO-CSIC), 28049 Madrid, Spain
${ }^{3}$ Unidad de Proteómica, Centro Nacional Investigaciones Cardiovasculares (CNIC), 28029 Madrid,
Spain
${ }^{4}$ Group of Molecular Microbiology, Instituto de Biologia Molecular e Celular, Universidade do Porto,
Porto, Portugal
${ }^{5}$ Institut Pasteur, Plate-forme PF1 Génomique, Département Génomes et Génétique, Paris, France
${ }^{6}$ Institute of Medical Microbiology, Justus-Liebig-University, Giessen, D-35392, Germany
${ }^{7}$ Unité des Interactions Bactéries-Cellules, Institut National de la Santé et de la Recherche
Médicale (INSERM) U604, Institut Pasteur, and the Institut Scientifique de Recherche
Agronomique (INRA) USC2020, Institut Pasteur, Paris F-75015, France

Bacteria of the genus Listeria contain the largest family of LPXTG surface proteins covalently anchored to the peptidoglycan. The extent to which these proteins may function or be regulated cooperatively is at present unknown. Because of their unique cellular location, we reasoned that distinct LPXTG proteins could act as elements contributing to cell wall homeostasis or influencing the stability of other surface proteins bound to peptidoglycan. To test this hypothesis, we used proteomics to analyse mutants of the intracellular pathogen Listeria monocytogenes lacking distinct LPXTG proteins implicated in pathogen-host interactions, such as $\operatorname{In}|\mathrm{A}, \operatorname{In}| \mathrm{F}, \operatorname{In}|\mathrm{G}, \operatorname{In}| \mathrm{H}$, InIJ, LapB and Vip. Changes in the cell wall proteome were found in in/G and vip mutants, which exhibited reduced levels of the LPXTG proteins InIH, Lmo0610, Lmo0880 and Lmo2085, all regulated by the stress-related sigma factor SigB. The ultimate basis of this alteration was uncovered by genome sequencing, which revealed that these in/G and vip mutants carried lossof-function mutations in the $r s b S, r s b U$ and $r s b V$ genes encoding regulatory proteins that control $\mathrm{SigB}$ activity. Attempts to recapitulate this negative selection of $\mathrm{SigB}$ in a large series of new in/G or vip mutants constructed for this purpose were, however, unsuccessful. These results indicate that inadvertent secondary mutations affecting SigB functionality can randomly arise in $L$. monocytogenes when using common genetic procedures or during subculturing. Testing of SigB activity could be therefore valuable when manipulating genetically $L$. monocytogenes prior to any subsequent phenotypic analysis. This test may be even more justified when generating deletions affecting cell envelope components.
Abbreviations: RT-PCR, reverse transcriptase PCR; SNPs, simple nucleotide polymorphisms.

Three supplementary figures and three supplementary tables are available with the online version of this paper.

\section{INTRODUCTION}

Listeria monocytogenes is a Gram-positive facultative intracellular bacterium responsible for food-borne infections in humans and animals. Upon infection, this pathogen crosses the intestinal, blood-brain and placental 
barriers leading to gastroenteritis, meningoencephalitis and materno-fetal infections in humans with a fatality rate estimated at $20-30 \%$ of infected individuals (Cossart \& Toledo-Arana, 2008; Hamon et al., 2006; Vázquez-Boland et al., 2001). Several surface proteins of this pathogen display distinct modes of association with the cell wall and direct key steps of the infection process, including bacterial adhesion to host cells, pathogen internalization and subversion of host cell cytoskeleton dynamics (Bierne \& Cossart, 2007). A remarkable feature shared by all species of the Listeria genus is the high content of genes encoding surface proteins which bear an LPXTG sorting motif (Bierne \& Cossart, 2007; Doumith et al., 2004; Glaser et al., 2001; Hain et al., 2007). This motif is present in more than 40 surface proteins of these bacteria and is recognized by sortases, specialized enzymes that mediate their covalent anchorage to the peptidoglycan (Marraffini et al., 2006; Spirig et al., 2011). How bacteria regulate the production of the set of LPXTG proteins is at present unknown, although recent data obtained in $L$. monocytogenes suggest that sortase A may be a contributing factor (Mariscotti et al., 2012). Coordinate regulation has been proposed for the production of different cell wall polymers, including peptidoglycan, teichoic acids, lipoteichoic acids and the capsule (Hanson \& Neely, 2012). Whether such a type of regulation occurs at the level of surface proteins that interact with the cell wall is at present unknown.

In this study, we report a cell wall proteome analysis of $L$. monocytogenes mutants lacking distinct LPXTG proteins such as InlA, InlF, InlG, InlH, Inl,, LapB and Vip, previously characterized for their contribution to virulence and to pathogen-host cell interactions (Cabanes et al., 2005; Guzman et al., 1995; Kirchner \& Higgins, 2008; Lingnau et al., 1995; Raffelsbauer et al., 1998; Reis et al., 2010; Sabet et al., 2005). Two of these mutants, $\Delta i n l G$ and $\Delta v i p$, inadvertently exhibited a defective proteome characteristic of a deficiency in LPXTG proteins regulated positively by the stress-related alternate sigma factor SigB. This sigma factor plays a central role in promoting adaptation to different types of stress and virulence in $L$. monocytogenes as well as other Gram-positive bacteria e.g. Bacillus subtilis and Staphylococcus aureus (Abram et al., 2008a; Chaturongakul \& Boor, 2006; Giotis et al., 2008; Kazmierczak et al., 2006; Palmer et al., 2009; Raengpradub et al., 2008; Swaminathan \& Gerner-Smidt, 2007). Further studies uncovered the presence of secondary mutations causing loss of function in regulatory elements required to activate SigB, such as RsbS, RsbU and RsbV. These proteins form part of a complex regulatory cascade starting in the membrane-bound stressosome protein complex, which triggers a signal transduction upon perception of the stress stimuli. Such a signal culminates with the phosphorylation of the anti-sigma protein RsbW and the release of SigB from the sigma/anti-sigma complex (O'Byrne \& Karatzas, 2008). Appearance of mutations affecting SigB activity was observed in independent $\Delta i n l G$ and $\Delta v i p$ mutants generated in different laboratories. Considering that SigB is an important factor mediating stress survival and virulence in Gram-positive bacterial pathogens (Cheung et al., 2004; Hecker et al., 2007; Novick, 2003; OByrne \& Karatzas, 2008), the results reported here highlight the value of monitoring activity of the regulon controlled by this protein when performing genetic manipulations in $L$. monocytogenes.

\section{METHODS}

Bacterial strains and culture conditions. The L. monocytogenes strain EGDe (Glaser et al., 2001) of serotype 1/2a was used as parental strain. The isogenic mutants lacking LPXTG proteins used in this study are listed in Table 1. Unless otherwise indicated, bacteria were grown in brain heart infusion (BHI) medium at $37{ }^{\circ} \mathrm{C}$ in shaking aerobic conditions.

Complementation of in/G and vip mutations. For complementation, inlG and vip alleles were PCR-amplified from chromosomal DNA of wt strain EGDe and cloned in the SacI and SphI restriction sites of the pP1 plasmid (Dramsi et al., 1995) using primers listed in Table S1 (available in Microbiology Online). The final constructs were verified by sequencing and the relative expression of the transgene estimated by reverse transcriptase PCR (RT-PCR) using oligonucleotides primers listed in Table S1.

Preparation of cell wall extract for proteomic analysis. Peptidoglycan was purified from wt and isogenic mutants lacking LPXTG proteins grown in BHI medium to exponential phase, as previously described (Calvo et al., 2005). Purified peptidoglycan was digested with modified trypsin (sequencing grade, Promega), as described previously (Calvo et al., 2005). The resulting peptide mixture was lyophilized and kept at $-20{ }^{\circ} \mathrm{C}$. Protein identification by liquid chromatography-tandem mass spectrometry was performed as described previously (García-del Portillo et al., 2011).

\begin{abstract}
Western blot analyses of cell surface proteins bound to peptidoglycan. Cell wall extracts were prepared from bacteria grown at $37{ }^{\circ} \mathrm{C}$ in $\mathrm{BHI}$ medium under non-shaking conditions, as described previously (Pucciarelli et al., 2005). To release covalently bound LPXTG proteins from cell walls, whole bacteria were incubated with mutanolysin to digest peptidoglycan (Pucciarelli et al., 2005). Polyclonal rabbit anti-Lmo0160, anti-Lmo0171, anti-InlG, antiInlH, anti-Vip, anti-Lmo0610, anti-Lmo0880 and anti-Lmo2085 immune sera were generated using full-length His-tagged recombinant proteins as antigens. Mouse monoclonal anti-InlA (Mengaud et al., 1996) was used as control for cell wall fractions. Goat anti-mouse and goat anti-rabbit antibodies conjugated to horseradish peroxidase (Bio-Rad) were used as secondary antibodies. Proteins were visualized by chemoluminescence using luciferin-luminol reagents.
\end{abstract}

RNA extraction and semiquantitative RT-PCR. RNA was purified from bacteria grown in $10 \mathrm{ml}$ of BHI at exponential phase $\left(\mathrm{OD}_{600}=0.2\right)$ using the TRIzol reagent method (Invitrogen), as described previously (Toledo-Arana et al., 2009). RNA integrity and concentration were assessed by agarose-Tris-acetate-EDTA electrophoresis and absorbance at $260 \mathrm{~nm}$ after treatment with DNase I for $30 \mathrm{~min}$ at $37^{\circ} \mathrm{C}$ (Turbo DNA-free kit, Ambion/Applied Biosystems). RT-PCR was performed using a one-step RT-PCR kit (Qiagen). Briefly, RT-PCR was carried out in a final volume of $25 \mu$ l consisting of $5 \mu \mathrm{l}$ buffer $(5 \times), 1 \mu \mathrm{l}$ of dNTP $(10 \mathrm{mM}), 3 \mu \mathrm{l}$ each of the forward and reverse primers $(5 \mathrm{mM}), 1 \mu \mathrm{l}$ of RT-PCR enzyme mix, $40 \mathrm{ng}$ of RNA and RNase-free water. RT-PCR cycling conditions were as follows: $50{ }^{\circ} \mathrm{C}$ for $35 \mathrm{~min}$ and $95{ }^{\circ} \mathrm{C}$ for $15 \mathrm{~min}$, followed by 30 cycles of $94{ }^{\circ} \mathrm{C}$ for $30 \mathrm{~s}, 55^{\circ} \mathrm{C}$ for $30 \mathrm{~s}$ and $72{ }^{\circ} \mathrm{C}$ for $1 \mathrm{~min}$, and then an 
Table 1. Listeria monocytogenes strains used in this study

\begin{tabular}{|c|c|c|}
\hline Strain & Relevant genotype & Source or reference \\
\hline EGDe & wt & Glaser et al. (2001) \\
\hline MD1297 & $\Delta i n l G(1)$ & This work \\
\hline MD3415 & $\Delta \operatorname{inl} G(2)$ & This work \\
\hline MD1660 & $\Delta i n l F$ & This work \\
\hline MD1658 & $\Delta \operatorname{sig} B$ & This work \\
\hline MD2306 & $\Delta i n l A$ & This work \\
\hline DC220 & $\Delta \operatorname{lmo0320}($ vip) (1) & This work \\
\hline DC431 & $\Delta l m o 0320$ (vip) (2) & This work \\
\hline BUG2235 & $\Delta \operatorname{lmo0320}($ vip $):: \mathrm{km}$ & Cabanes et al. (2005) \\
\hline MD2701 & $\Delta l m o 0320$ (vip) (1)pP1lmo320 & This work \\
\hline MD2708 & $\Delta l m o 0320$ (vip) (1)pP1rsbV & This work \\
\hline MD2709 & $\Delta l m o 0320$ (vip):: $\mathrm{km} \mathrm{pP1rsbS}$ & This work \\
\hline MD2711 & EGD-epP1lmo320 & This work \\
\hline MD2712 & $\Delta i n l G(1) \mathrm{pP} 1 i n l G$ & This work \\
\hline DC109 & $\Delta l a p B$ & Reis et al. (2010) \\
\hline BUG2569 & $\Delta i n l H$ & Personnic et al. (2010) \\
\hline BUG2159 & $\Delta$ inlJ & Sabet et al. (2005) \\
\hline
\end{tabular}

extra elongation step at $72{ }^{\circ} \mathrm{C}$ for $10 \mathrm{~min}$. Oligonucleotides used in these RT-PCR assays were designed using the software Primer Express v3.0 (Applied Biosystems) and are listed in Table S1.

Nucleotide sequencing of rsbRSTU and rsbVW-sigB-rsbX operons in $\Delta i n / G, \Delta v i p$ and $\Delta v i p::$ km mutants. Sequencing of the $r s b R S T U$ and $r s b V W$-sigB-rsbX operons was performed upon PCR amplification of 500-600 bp fragments from chromosomal DNA isolated from wt EGDe strain, the original mutant $\Delta v i p:: \mathrm{km}$ (Cabanes et al., 2005), and the $\Delta v i p(1)$ and $\Delta i n l G(1)$ deletion mutants constructed for this study. Oligonucleotides used are listed in Table S1.

Whole-genome sequencing. Chromosomal DNA from L. monocytogenes EGDe, $\Delta v i p:: \mathrm{km}$ (Cabanes et al., 2005), $\Delta v i p$ and $\Delta i n l G$ strains was extracted using cetyl-trimethyl-ammonium bromide and alkaline lysis, as described previously (Wilson, 2001). Bacteria were previously treated with $20 \mathrm{mg} \mathrm{ml}^{-1}$ lysozyme for $20 \mathrm{~min}$ at $37{ }^{\circ} \mathrm{C}$ in Tris-EDTA-sucrose buffer. Samples were further treated with $0.5 \mathrm{mg}$ $\mathrm{ml}^{-1}$ RNase A (bovine pancreas, Roche) for $30 \mathrm{~min}$ at $37^{\circ} \mathrm{C}$ and the same volume of phenol was added. Chromosomal DNA was finally precipitated with 2-propanol, washed in $70 \%(\mathrm{v} / \mathrm{v})$ ethanol and airdried before suspending in MiliQ-filtered water. Absence of contaminating RNA was tested in $0.8 \%$ agarose gels and by measurement of the $A_{260} / A_{280}$ absorbance ratio, which was in all samples approx. 2.0. Whole-genome sequencing of strains were performed with a single-reads sequencing technology. Illumina library preparation and sequencing followed standard protocols developed by the supplier. Single reads of 100 cycles were collected on a HiSeq 2000 (Illumina). After sequencing was complete, image analysis, base calling and error estimation were performed using Illumina Analysis Pipeline version 1.7.

Construction of a new collection of $L$. monocytogenes $\Delta$ in/G and $\Delta$ vip deletion mutants. To construct the $\Delta i n l G$ mutant strain, fragments containing $\sim 500$ bp DNA flanking the ORFs of inlG (Imo0262) were amplified by PCR using chromosomal DNA of $L$. monocytogenes strain EGD-e and cloned into the suicide integrative vector pAUL-A, as previously described (Chakraborty et al., 1992; Dussurget et al., 2002). Oligonucleotide primers used are listed in Table S1. To construct the $\Delta v i p$ mutant strain, we used a modified version of pDC4 (Cabanes et al., 2005). The vector was digested with $K p n \mathrm{I}$ and $\mathrm{XbaI}$ to remove the kanamycin resistance cassette, treated with T4 DNA polymerase, religated and transformed into E. coli XL1Blue to yield pDC218. Gene deletion by double recombination was performed as described previously (Arnaud et al., 2004; Cabanes et al., 2004; Wouters et al., 2005). These original mutants lacking the ORFs of $i n l G$ and $v i p$ were referred as series '1', i.e. $\Delta i n l G(1)$ and $\Delta v i p(1)$. A different $\Delta v i p$ deletion mutant, named $\Delta v i p(2)$, was also generated leaving $63 \mathrm{nt}$ in the $5^{\prime}$ and $30 \mathrm{nt}$ in the $3^{\prime}$ terminal ends of the vip gene. Fragments containing $\sim 1000$ bp DNA flanking the ORFs of vip were amplified by PCR using chromosomal DNA of L. monocytogenes strain EGDe. Oligonucleotide primers used are listed in Table S1. These $5^{\prime}$ and $3^{\prime}$ flanking $\sim 1000$ bp fragments were digested with SalI/ $M l u \mathrm{I}$ and $M l u \mathrm{I} / N c o \mathrm{I}$ respectively and cloned into the suicide integrative vector pMAD to yield pDC425 (Arnaud et al., 2004). Gene deletion by double recombination was performed as described previously (Boneca et al., 2007). Likewise, a second $\Delta i n l G(2)$ was also generated by gene splicing by overlap extension (gene SOEing) and a deletion protocol based in the pAUL-A. Gene replacement was performed as described previously (Arnaud et al., 2004; Cabanes et al., 2004; Wouters et al., 2005) but the non-permissive temperature was changed to $42{ }^{\circ} \mathrm{C}$. The $\Delta i n l G(1), \Delta i n l G(2), \Delta v i p(1)$ and $\Delta v i p(2)$ deletions were verified by PCR using the primers listed in Table S1. The comparison among the protocols used is shown in Table S2.

Evaluation of SigB function by chitinase activity. The functional status of the SigB regulon was tested by monitoring chitinase activity in bacteria grown on solid medium containing tryptone $\left(10 \mathrm{~g} \mathrm{l}^{-1}\right)$, yeast extract $\left(5 \mathrm{gl}^{-1}\right), \mathrm{NaCl}\left(10 \mathrm{gl}^{-1}\right)$, agar $\left(15 \mathrm{~g} \mathrm{l}^{-1}\right)$ and phosphate buffer, pH 6.9 (Oxoid). This medium was supplemented with acid hydrolysed chitin $\left(3.0 \mathrm{~g} \mathrm{l}^{-1}\right)$ as described previously (Larsen et al., 2010), omitting the freeze-drying steps. The plates were incubated under aerobic conditions at $30{ }^{\circ} \mathrm{C}$ and scored for chitinase activity (clearing zones) for 5 days.

\section{RESULTS}

\section{Cell wall proteomics of $L$. monocytogenes mutants deficient in LPXTG surface proteins}

Cooperative regulation is known to exist among cell wall components of Gram-positive bacteria such as peptidoglycan, 
capsule, lipoteichoic and teichoic acids (Hanson \& Neely, 2012). Thus, the absence of one of these cell wall components can affect the production, stability or modification of the others. For example, disruption of teichoic acid production in S. aureus alters peptidoglycan cross-linkage and the activity of some autolysins (Atilano et al., 2010; Schlag et al., 2010). Based on these observations, we reasoned that some members of the family of LPXTG surface proteins of L. monocytogenes could contribute to cell wall homeostasis and influence the relative amount of other cell wall components, including surface proteins strongly bound to peptidoglycan. To test this hypothesis, we used high-resolution MS to analyse the protein content of cell wall extracts prepared from L. monocytogenes EGDe-derived mutants lacking LPXTG proteins. As most of the L. monocytogenes genes encoding LPXTG proteins are still uncharacterized, we examined previously reported mutants lacking LPXTG proteins involved in virulence and pathogenhost cell interactions. These mutants included $\triangle i n l A, \triangle i n l F$, $\Delta$ inlG, $\Delta i n l H, \Delta i n l J, \Delta l a p B$ and $\Delta v i p$ (Cabanes et al., 2005; Guzman et al., 1995; Kirchner \& Higgins, 2008; Lingnau et al., 1995; Personnic et al., 2010; Raffelsbauer et al., 1998; Reis et al., 2010; Sabet et al., 2005). High-resolution MS analysis revealed that the cell wall proteome of most of these mutants remained essentially unchanged with respect to the content of LPXTG proteins. Some minor changes were observed for Lmo0171, an LPXTG protein identified with an average of four unique peptides, except in the $\Delta i n l F$ and $\Delta i n l H$ strains (Table 2); and InlG, identified with an average of six peptides, except in the $\Delta$ inlA strain (Table 2). These differences were, however, not confirmed by Western blotting using cell wall extracts of these strains and specific anti-Lmol71 and antiInlG antibodies (Fig. S1). This finding was indicative of the difficulty of identifying minor changes in the relative amount of low-abundance surface proteins. In sharp contrast to the wt strain, no peptides corresponding to the LPXTG proteins Lmo0263 (InlH), Lmo0610, Lmo0880 and Lmo2085 were detected in the cell wall of the $\Delta i n l G$ and $\Delta v i p$ mutants (Table 2). Of interest, the genes encoding these four LPXTG proteins are regulated by the stress-related sigma factor SigB (Hain et al., 2008; Loh et al., 2006). This observation led us to postulate that InlG and Vip could contribute to cell wall homeostasis and that these two LPXTG proteins could work with SigB for the correct sensing of stresses linked to cell wall alterations. Alternatively, the absence on the bacterial surface of InlG or Vip could disrupt cell wall homeostasis and, as a consequence, generate a stress that could overstimulate SigB resulting in a deleterious effect to the cell. In this latter scenario, loss-offunction mutations abrogating SigB function were expected to be selected.

\section{Validation of proteomic data uncovers the presence of additional mutations affecting SigB function in $\Delta i n / G$ and $\Delta v i p$ mutants}

Western blots were performed to validate the proteomic data obtained for the $\Delta i n l G$ and $\Delta v i p$ mutants by an alternative method. A $\Delta \operatorname{sig} B$ mutant was also included as control. These experiments confirmed that the lack of InlG or Vip correlated with a dramatic decrease of SigBregulated proteins such as $\mathrm{InlH}$, Lmo0610 and $\mathrm{Lmo} 0880$ (Fig. 1a) in the cell wall. This effect was specific since it was not observed for other LPXTG proteins, such as Lmo0160 or surface proteins strongly bound to the peptidoglycan by the LysM domain, e.g. the autolysin P60 (Fig. 1a). To determine whether these changes reflected a failure of the entire SigB regulon, we monitored the expression of genes positively regulated by SigB but not related to cell wall such as bsh and lmo2695, which encode a bile salt hydrolase and a subunit of the dihydroxyacetone kinase enzyme, respectively (Abram et al., 2008b; Begley et al., 2005). Reverse transcriptase PCR (RT-PCR) assays confirmed that, like the phenotype observed in the $\Delta \operatorname{sig} B$ mutant, expression of bsh and $l m o 2695$ was reduced in the $\Delta i n l G$ and $\Delta v i p$ mutants (Fig. 1b). Decreased amounts of $\operatorname{sig} B$ transcript and SigB protein were evident in the inl $G$ and $\Delta v i p$ mutants (Fig. 1b), which was consistent with the lower activity of the SigB regulon in these two mutants and the positive auto-regulation that $\mathrm{SigB}$ exerts over the $r s b \mathrm{~V}$ $r s b W$-sigB-rsbX regulon (O'Byrne \& Karatzas, 2008). Similar findings were obtained with a previously reported $\Delta v i p:: \mathrm{km}$ mutant (Cabanes et al., 2005), which also turned out to be defective in the production of LPXTG proteins regulated positively by SigB (data not shown). Furthermore, all the three mutants, $\Delta i n l G, \Delta v i p$ and $\Delta v i p:: \mathrm{km}$, displayed sensitivity to acid stress in a similar fashion to the $\Delta \operatorname{sig} B$ mutant (Fig. S2). Taken together, these observations indicated that $\mathrm{SigB}$ function was dramatically impaired in the L. monocytogenes lacking the LPXTG proteins InlG or Vip. Unexpectedly, complementation assays with wt $i n l G^{+}$and $v_{i p}{ }^{+}$alleles did not result in suppression of any of the phenotypes related to the loss-offunction in SigB. As a representative example, recovery in the production of the SigB-regulated LPXTG protein Lmo0880 was not observed in the complemented strains (Fig. 1c). These strains were also found to be impaired in the production of other SigB-regulated LPXTG proteins, as InlH and Lmo0610 (data not shown). Expression of the wt $v i p^{+}$allele in the $\Delta v i p:: \mathrm{km}$ mutant also failed to restore SigB functionality (data not shown). Altogether, these findings supported the existence of additional secondary mutations present in the $\Delta i n l G$ and $\Delta v i p$ mutants that were affecting $\operatorname{SigB}$ functionality. Since the SigB protein was detected at its predicted molecular mass in the $\Delta i n l G$ and $\Delta v i p$ mutants (Fig. 1b), such loss of function could be tentatively be assigned to point mutations in the $\operatorname{sig} B$ gene resulting in a non-functional protein. Additionally, there are proteins that form the regulatory cascade that modulate SigB's activity (O'Byrne \& Karatzas, 2008). SigB function is primarily regulated at the level of free protein that remains unbound from its anti-sigma factor, RsbW. Formation of the SigB-RsbW complex depends on the phosphorylation status of RsbW (O'Byrne \& Karatzas, 2008). Thus, RsbW acquires auto-kinase activity upon binding to RsbV, a process that results in phosphorylated RsbW that dissociates from SigB. Mutations affecting either RsbW or upstream elements of the regulatory cascade that determine 
Table 2. Surface proteins covalently bound to peptidoglycan identified by high resolution MS in the cell wall of $L$. monocytogenes mutants lacking LPXTG proteins involved in virulence

\begin{tabular}{|c|c|c|c|c|c|c|c|c|c|}
\hline \multirow[b]{2}{*}{ Surface protein $\dagger$} & \multirow[b]{2}{*}{ Function } & \multicolumn{8}{|c|}{ Unique peptides identified per protein and mutant ${ }^{\star}$} \\
\hline & & EGDe (wt) & $\Delta i n l A$ & $\Delta i n l F$ & $\Delta i n l G$ & $\Delta i n l H$ & $\Delta i n l J$ & $\Delta l a p B$ & $\Delta v i p$ \\
\hline Lmo2185 & Unknown, sortase-B substrate & 41 & 31 & 38 & 32 & 42 & 45 & 44 & 48 \\
\hline Lmo2186 & Unknown, sortase-B substrate & 9 & 8 & 10 & 10 & 11 & 9 & 13 & 13 \\
\hline Lmo2714 & Peptidoglycan anchored protein (LPXTG motif) & 17 & 14 & 17 & 18 & 16 & 15 & 25 & 17 \\
\hline Lmo0130 & $\begin{array}{l}\text { Similar to } 5 \text {-nucleotidase, putative peptidoglycan bound protein } \\
\text { (LPXTG motif) }\end{array}$ & 31 & 24 & 39 & 35 & 31 & 32 & 29 & 35 \\
\hline Lmo0880 & Similar to cell surface proteins (LPXTG motif) & 18 & 9 & 15 & - & 20 & 17 & 20 & - \\
\hline Lmo1666 (LapB) & LPXTG protein adhesin required for entry into eukaryotic cells & 20 & 15 & 23 & 21 & 25 & 23 & $(\ddagger)$ & 22 \\
\hline Lmo0160 & Putative peptidoglycan bound protein (LPXTG motif) & 19 & 15 & 22 & 15 & 19 & 17 & 18 & 18 \\
\hline Lmo0433 (InlA) & Internalin A (InlA); invasin & 19 & $(\ddagger)$ & 13 & 10 & 18 & 20 & 18 & 10 \\
\hline Lmo0263 (InlH) & Internalin $\mathbf{H}(\mathbf{I n l H})$ & 10 & 8 & 11 & - & $(\ddagger)$ & 13 & 16 & - \\
\hline Lmo2178 & Putative peptidoglycan bound protein (LPXTG motif) & 10 & 9 & 8 & 9 & 8 & 15 & 16 & 12 \\
\hline Lmo0514 & $\begin{array}{l}\text { Similar to internalin proteins, putative peptidoglycan bound protein } \\
\text { (LPXTG motif) }\end{array}$ & 10 & 7 & 10 & 6 & 5 & 9 & 13 & 7 \\
\hline Lmo0842 & Putative peptidoglycan bound protein (LPXTG motif) & 10 & 9 & 13 & 4 & 8 & 8 & 9 & 10 \\
\hline Lmo0327 & Similar to cell surface proteins (LPXTG motif) & 8 & 6 & 11 & 15 & 6 & 9 & 6 & 11 \\
\hline Lmo0610 & $\begin{array}{l}\text { Similar to internalin proteins, putative peptidoglycan bound } \\
\text { protein (LPXTG motif) }\end{array}$ & 7 & 4 & 11 & - & 9 & 7 & 6 & - \\
\hline Lmo0262 (InlG) & Internalin G (InlG) & 5 & - & 3 & $\ddagger$ & 6 & 7 & 7 & 5 \\
\hline Lmo1413 & $\begin{array}{l}\text { Similar to internalin, putative peptidoglycan bound protein (LPXTG } \\
\text { motif) }\end{array}$ & 4 & 6 & - & 2 & 3 & 3 & 4 & 3 \\
\hline Lmo2085 & Putative peptidoglycan bound protein (LPXTG motif) & 2 & 2 & 6 & - & 8 & 6 & 7 & - \\
\hline Lmo0171 & $\begin{array}{l}\text { Similar to internalin proteins, putative peptidoglycan bound protein } \\
\text { (LPXTG motif) }\end{array}$ & 5 & 4 & - & 4 & - & 4 & 7 & 3 \\
\hline Lmo2396 & $\begin{array}{l}\text { Similar to internalin proteins, putative peptidoglycan bound protein } \\
\text { (LPXTG motif) }\end{array}$ & 1 & 1 & 2 & 3 & 2 & 4 & 3 & 2 \\
\hline Lmo0835 & Putative peptidoglycan bound protein (LPXTG motif) & - & - & 9 & 3 & - & 1 & - & 1 \\
\hline Lmo2026 & Putative peptidoglycan bound protein (LPXTG motif) & 2 & 2 & - & 2 & 2 & 2 & 1 & 2 \\
\hline Lmo0159 & Putative peptidoglycan bound protein (LPXTG motif) & 3 & 2 & - & 2 & 5 & 2 & 4 & 3 \\
\hline Lmo0331 & $\begin{array}{l}\text { Similar to internalin, putative peptidoglycan bound protein (LPXTG } \\
\text { motif) }\end{array}$ & 1 & - & - & 2 & - & 2 & 3 & 1 \\
\hline Lmo1290 & $\begin{array}{l}\text { Similar to internalin, putative peptidoglycan bound protein (LPXTG } \\
\text { motif) }\end{array}$ & 2 & - & - & - & 2 & 2 & 1 & 1 \\
\hline Lmo2821 (InlJ) & Internalin J (InlJ) & 1 & - & - & 2 & - & $(\ddagger)$ & - & 2 \\
\hline Lmo0333 & Putative peptidoglycan bound protein (LPXTG motif) & 1 & - & - & 1 & - & - & - & - \\
\hline Lmol136 & $\begin{array}{l}\text { Similar to internalin, putative peptidoglycan bound protein (LPXTG } \\
\text { motif) }\end{array}$ & - & 1 & - & - & - & - & - & - \\
\hline
\end{tabular}


the ratio of phosphorylated to non-phosphorylated RsbW could also have similar consequences. For example, a null mutation in RsbV could result in a shift of all RsbW molecules to the non-phosphorylated form and, as a consequence, a long-term state of SigB in its inactive state trapped with the anti-sigma factor.

\section{Mapping of the mutations carried by $\Delta i n / G$ and $\Delta v i p$ mutants affecting SigB function}

To discern the type of mutations carried by the $\Delta i n l G, \Delta v i p$ and $\Delta v i p:: \mathrm{km}$ mutants that were impairing SigB function, we first considered their presence in $\operatorname{sig} B$ itself or some of the genes encoding elements forming the regulatory cascade that controls RsbW phosphorylation. These elements, together with RsbW and SigB itself, are encoded in the $r s b R S T U$ and $r s b V W$-sigB-rsbX operons. Specific oligonucleotides were designed to obtain the complete nucleotide sequence of all ORFs in the two operons and their respective promoter regions (Table S1). This sequencing showed the presence of loss-of-function mutations in each of the $\Delta i n l G, \Delta v i p:: \mathrm{km}$ and $\Delta v i p$ mutants affecting distinct elements of the regulatory cascade (Fig. 2a). More precisely, the $\Delta$ inl $G$ mutant harbours an $\mathrm{A} \rightarrow-/ \mathrm{A}$ frame shift in the $r s b U$ coding sequence that disrupts the protein from amino acid residue 195, while the $\Delta v i p:: \mathrm{km}$ mutant contains a nonsense mutation CAG (Gln) $\rightarrow$ TAG (stop) of the $r s b S$ coding region corresponding to codon of amino acid residue 17. In the $\Delta v i p$ mutant, herein referred to as $\Delta v i p(1)$ to distinguish it from the published $\Delta v i p:: \mathrm{km}$ mutant (Cabanes et al., 2005), a nonsense mutation GAA (Glu) $\rightarrow$ TAA (stop) was found in the coding region of $r s b V$ corresponding to codon of amino acid residue 9 (Fig. 2a). To further confirm that these mutations were responsible for the lack of SigB function, we selected the $\Delta v i p:: \mathrm{km}$ and $\Delta v i p$ mutants for complementation assays with the respective $r s b S^{+}$and $r s b V^{+}$wild-type alleles. Expression of the $r s b V^{+}$allele in the $\Delta v i p(1)$ mutant restored the production of SigB-regulated LPXTG proteins such as InlH, Lmo0880 or Lmo0610 (Fig. 2b). However, no such effect was observed for the $\Delta v i p:: \mathrm{km} / r s b S^{+}$complemented strain (Fig. 2b). Unlike RsbV, which is a cytosolic protein, RsbS is part of the stressosome complex. This complex remains uncharacterized in L. monocytogenes but is believed to function as in B. subtilis. The stressosome acts in the envelope to perceive external environmental signals that stimulate a signal transduction cascade which activates SigB upon release of its cognate anti-sigma factor (O’Byrne \& Karatzas, 2008). Interestingly, RsbS is phosphorylated upon signal perception. Based on this, it is possible that RsbS overexpression occurring in our complemented $\Delta v i p:: \mathrm{km} / r s b S^{+}$strain may lead to changes in the stoichiometry of the stressosome affecting the phosphorylation step. Likewise, our findings did not allow us to discard the possibility that the $\Delta v i p:: \mathrm{km}$ mutant could carry additional mutations besides that found in $r s b S$ (Fig. 2a). Similar negative results were obtained when attempting to complement the $\Delta i n l G$ mutant with an 

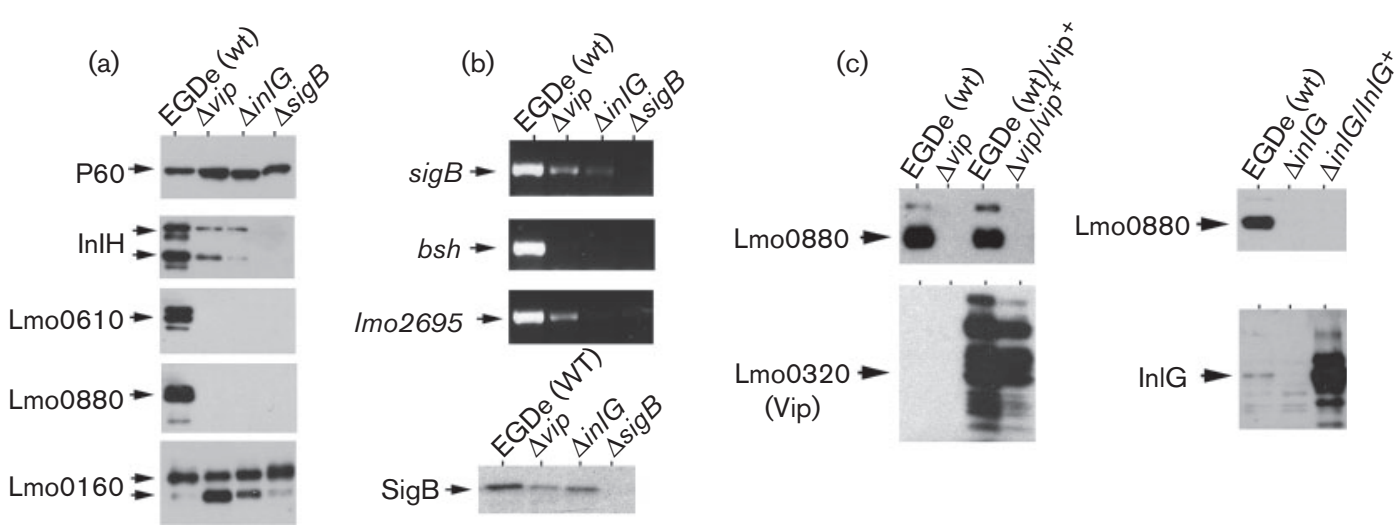

Fig. 1. L. monocytogenes mutants defective in the LPXTG surface proteins InIG or Vip display deficient function of the SigB regulon. (a) Levels of individual surface proteins detected in cell wall extracts prepared from the indicated strains. Among the proteins shown, InlH, Lmo0610, and Lmo0880 are regulated by SigB. (b) Lack of either InlG or Vip affects the entire SigB regulon as shown by the reduced expression of SigB-regulated genes not related to cell wall metabolism, such as bsh and Imo2695. (c) Expression in trans of wt in/G ${ }^{+}$and vip ${ }^{+}$alleles does not restore SigB functionality in the $\Delta i n / G$ and $\Delta v i p$ mutants.

$r s b U^{+}$allele (data not shown). Complementation based on exchange of the $r s b U$ and $r s b V$ mutant alleles by wild-type sequences could have proved that these were the only causative mutations for the loss of activity in the SigB regulon. However, allelic exchange methods involve genetic manipulations that include in some cases integration and excision of suicide plasmids into the chromosome. Given the nature of our study, we avoided this subsequent

(a)

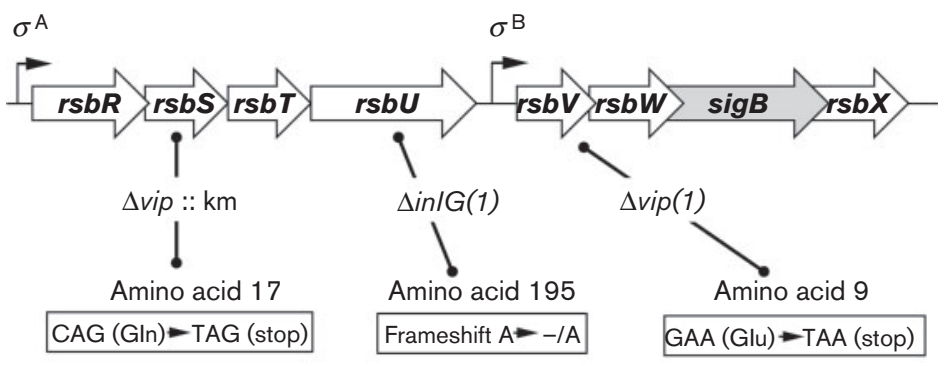

(b)

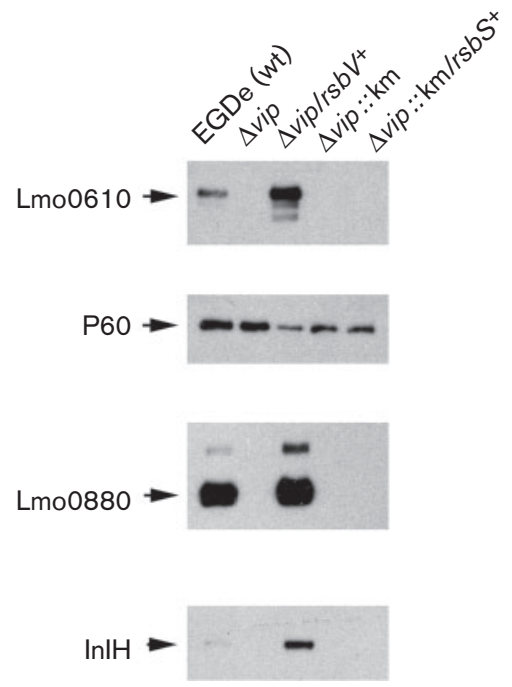

Fig. 2. Independent $\Delta i n / G$ and $\Delta v i p$ mutants counter-selected SigB functionality by mutations in genes encoding elements of the SigB regulatory cascade. (a) Mutations uncovered by sequencing of the rsbRSTU and rsbVWsigB-rsbX operons with specific oligonucleotides. Note that in each case the indicated gene products are lost by either nonsense mutations or a frameshift in the respective ORFs. (b) Recovery of SigB function in the $\Delta v i p$ mutant upon expression of the $r s b V^{+}$ allele. Such complementation was not successful in the case of the $\Delta v i p:: \mathrm{km} / r s b S^{+}$ strain (see text for details). 
procedure to minimize the possibility of introducing new and unknown selective pressures in the original $\Delta v i p$ and $\Delta$ inl $G$ mutants. To discern whether additional mutations besides those found in the SigB-related operons could be present in these mutants, we accomplished complete genome sequencing of EGDe (wild-type), $\Delta i n l G, \Delta v i p:: \mathrm{km}$ and $\Delta v i p(1)$ strains. This procedure was facilitated by the known genome sequence of the EGDe strain (Glaser et al., 2001). A few discrepancies in the reads were found in lmo1799 and $l m o 0412$, two genes having a large number of internal nucleotide repeats and encoding an LPXTG protein and a protein of unknown function, respectively. Besides these ambiguities, genome comparison confirmed the deletions generated in the inlG and vip genes and each of the point mutations in $r s b$ genes previously identified by sequencing of the $r s b R S T U$ and $r s b V W$-sigB-rsbX operons (see above and Table S3). A few simple nucleotide polymorphisms (SNPs) were also found in intergenic regions and as missense mutations in coding regions of the Imo0247, Imo0319 and Imo2576 genes. Additional changes of the type Pro $\rightarrow$ Ala and Ala $\rightarrow$ Ser were found in the trpS and $u v r B$ genes of the $\Delta i n l G$ mutant as well as a nucleotide change of the type $\mathrm{G} \rightarrow \mathrm{T}$ in the $\Delta i n l G$, $\Delta v i p:: \mathrm{km}, \Delta v i p(1)$ mutants that was mapping in the terminator region of the $r p m H$ gene encoding the $50 \mathrm{~S}$ ribosomal protein L34. These changes are summarized in Table S3. A closer analysis of these findings indicated that no genuine changes could be assigned to the $\Delta v i p:: \mathrm{km}$ mutant, which could not be complemented by the expression in trans of the $r s b S^{+}$gene, in comparison to the $\Delta v i p(1)$ mutant, which recovered SigB functionality upon complementation with the $r s b V^{+}$allele (Fig. 2b). Therefore, it was concluded that these defective mutants carried no additional changes affecting SigB functionality besides those mutations mapping in the rsb genes. Based on this, we reasoned that in those cases in which a lack of complementation was observed it could be due to the excess of protein expressed from the plasmid impairing proper signalling through the SigB regulatory cascade.

\section{Population study of the negative selection of SigB activity upon deletion of in/G or vip genes}

The $\Delta v i p:: \mathrm{km}, \Delta v i p(1)$ and $\Delta i n l G$ mutant strains described in previous experiments were constructed independently in three different laboratories. To further confirm that SigB function was counter-selected in bacteria lacking either InlG or Vip proteins, we generated two new $\Delta v i p$ and $\Delta$ inlG deletion mutants using similar genetic procedures involving a suicide integrative plasmid carrying flanking regions of the gene to be deleted (see Methods for details and Table S2). These new mutants were designated $\Delta v i p(2)$ and $\Delta i n l G(2)$. Strikingly, Western assays revealed that $\Delta v i p(2)$ and $\Delta i n l G(2)$ strains produced SigB-regulated LPXTG proteins InlH, Lmo0610, Lmo0880 and Lmo2085 at levels comparable to those of the wt strain (Fig. S3a, b). These observations raised the possibility that the original mutants $\Delta v i p:: \mathrm{km}, \Delta v i p(1)$ and $\Delta i n l G$ [hereafter named $\Delta i n l G(1)]$ were subjected to an unknown type of pressure during subculturing in different host laboratories or during the genetic manipulation that could result in counterselection of SigB function. Alternatively, some side effects related to the distinct plasmids used in the different genetic procedures could be responsible for the appearance of mutations abrogating SigB function. Finally, we considered the possibility that mutations affecting SigB function might occur stochastically and that the few $\Delta v i p$ and $\Delta i n l G$ mutants examined so far were not representative on a population basis. To test this, we generated a large collection of new $\Delta v i p$ and $\Delta i n l G$ mutants using the same plasmids that were used originally for constructing the $\Delta v i p(1), \Delta v i p(2)$ and $\Delta i n l G(2)$ strains (see Table S2). A total of 80,136 and 54 new clones were generated after the recombination events with distinct suicide plasmids (Table $3)$. PCR assays confirmed that 23, 23 and 15 mutants of each of these series were genuine $\Delta v i p$ and $\Delta i n l G$ deletion mutants (Table 3). To assess SigB functionality in these clones, we applied a rapid screening on plates based on monitoring of chitinase activity. Chitinase production is regulated positively by SigB (Larsen et al., 2010) and can be visualized as a halo surrounding bacteria growing on solid

Table 3. Series of new L. monocytogenes mutants defective in the LPXTG surface proteins Vip or InIG that were constructed and analysed for SigB function

\begin{tabular}{|c|c|c|c|}
\hline \multirow[t]{2}{*}{ PCR result in region of interest ${ }^{*}$} & \multicolumn{3}{|c|}{$\begin{array}{l}\text { Number of new clones obtained upon applying gene deletion procedures in the } \\
\text { L. monocytogenes genes vip and inlG }\end{array}$} \\
\hline & Series $\Delta v i p(3) \dagger$ & Series $\Delta v i p(4) \ddagger$ & Series $\Delta i n l G(3) \S$ \\
\hline wt & 57 & 113 & 39 \\
\hline Deletion mutation & 23 & 23 & 15 \\
\hline Total & 80 & 136 & 54 \\
\hline
\end{tabular}

${ }^{\star}$ See Fig. 3 for representative examples.

$\dagger$ Plasmid pDC218 (pKSV7 derivative), used originally to construct $\Delta v i p(1)$, was used to generate series $\Delta v i p(3)$.

$\ddagger$ Plasmid pDC425 (pMAD derivative), used originally to construct $\Delta v i p(2)$, was used to generate series $\Delta v i p(4)$.

§Plasmid pAUL-A, used originally to construct $\Delta i n l G(2)$, was used to generate series $\Delta i n l G(3)$. 
(a)

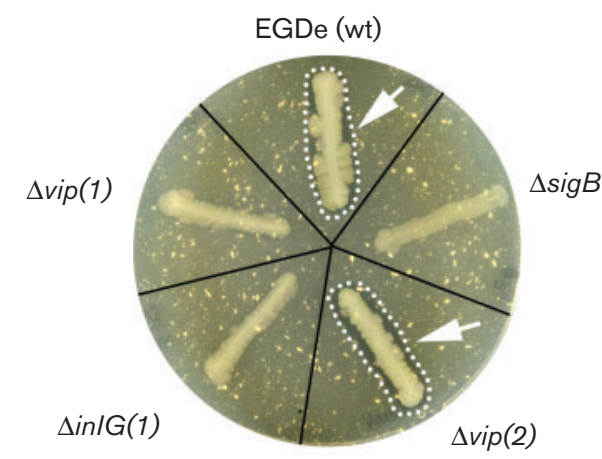

(b)
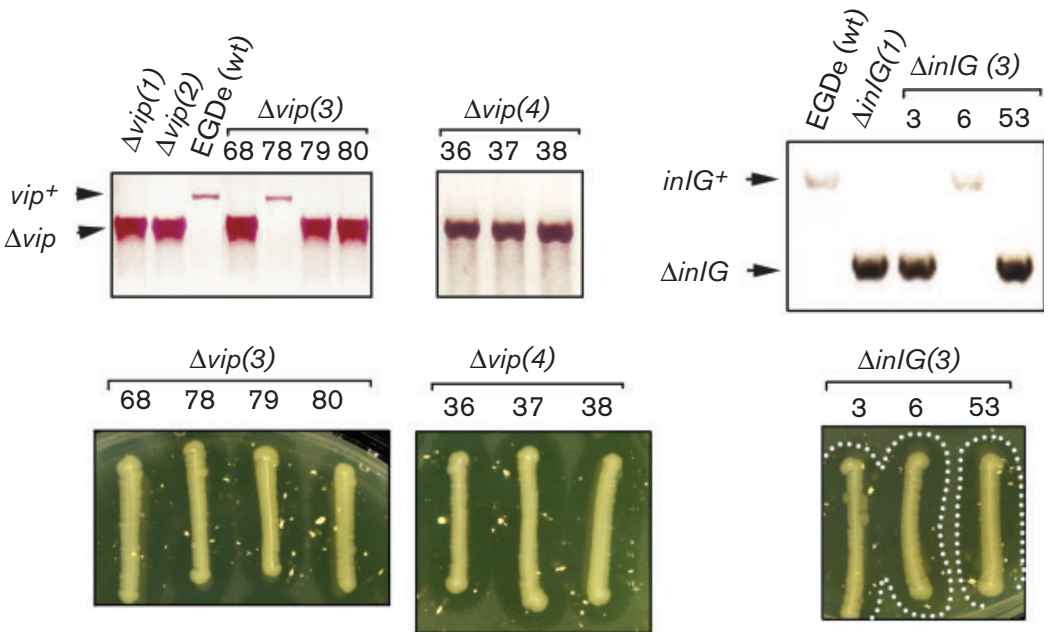

Fig. 3. Counter-selection of SigB function is not reproduced in a new series of $\Delta i n / G$ and $\Delta v i p$ deletion mutants generated with the same plasmid as the original mutant strains. (a) Screening of chitinase activity differentiates defective strains with no SigB function, such as $\Delta i n / G(1), \Delta v i p(1)$ and $\Delta s i g B$. Although not shown, the $\Delta v i p:$ : km mutant also exhibited no chitinase activity. Note that the second $\Delta v i p(2)$ mutant generated for this study behaved as wt bacterium concerning chitinase activity. Arrows and dotted lines indicate the clearing zones denoting such enzymatic activity. (b) Representative cases of clones generated upon usage of the suicide plasmids pAUL-A, pOD23 and pMAD (see Table 3 for details). Clones shown were obtained after resolution of the plasmids and tested by PCR. Note that regardless of the presence/absence of the corresponding in/G or vip genes, all clones displayed chitinase activity and therefore had a functional SigB regulon. The new series of mutants were named $\Delta i n / G(3), \Delta v i p(3)$ and $\Delta v i p(4)$ to differentiate them from the individual mutants tested previously (see text and Table 3 for details). Numbers identify individual clones of the three series.

media containing chitin. Control experiments showed that, unlike the wt bacteria and the $\Delta v i p(2)$ mutant, the $\Delta v i p(1)$, $\Delta i n l G(1)$ and $\Delta \operatorname{sig} B$ mutants were unable to degrade chitin (Fig. 3a). This result was consistent with the previous biochemical and proteomic analysis that unravelled the loss of SigB function. Surprisingly, none of the 61 deletion mutants generated ad hoc for this study $(23+23=46 \Delta v i p$ and $15 \Delta i n l G)$ was impaired in chitinase production. Some representative examples are shown in Fig. 3b. Additional phenotypic assays, such as sensitivity to an acidic environment ( $\mathrm{pH} 2.5$ ), confirmed that the newly generated series of $\Delta v i p$ and $\Delta i n l G$ mutants were all phenotypically $\mathrm{SigB}^{+}$(data not shown). Considering that this population study was performed on a significant number of $\Delta v i p$ and $\Delta$ inlG mutants, it seems probable that the counterselection of SigB function in the original mutants lacking the LPXTG surface proteins InlG or Vip was an event that occurred inadvertently.

\section{DISCUSSION}

This study was designed to dissect whether LPXTG surface proteins could contribute to maintenance of cell wall homeostasis in L. monocytogenes. The proteomic analysis uncovered an unexpected functional association between the LPXTG proteins InlG and Vip and the stress-related sigma factor SigB. Among the LPXTG protein-defective mutants generated for this study, those lacking InlG or Vip exhibited a marked decrease in the activity of the entire SigB operon. This finding was corroborated with another vip mutant previously reported to be affected in virulence (Cabanes et al., 2005). Surprisingly, counter-selection of SigB function following the deletion of inlG or vip genes was not confirmed in subsequent experiments in which a large number of new mutants were constructed. Unfortunately, no other mutant clones were kept in collections from the original knock-out experiments, so we are uncertain of the ultimate reasons why such mutations causing loss-of-function in SigB emerged in those initial genetic manipulations. The possibility that such counter-selection occurred inadvertently during subculturing in different host laboratories should also be considered. Although spontaneous mutations affecting sigma factors have been previously described in the literature, this report is the first that correlates such negative selection to the probable generation of stress linked to the alteration of cell wall-associated proteins. In Gram-negative bacteria like Escherichia coli, stress resistance varies between strains, suggesting that mutations favouring increased stress resistance may be counterselected in certain environments (Ferenci, 2008). Of interest, no correlation has been found between counterselection of the alternative sigma factor RpoS, required to cope with nutritional stress, and its expression level in different environments (King et al., 2006). Based on this, it has been suggested that mutational rpoS sweeps could be 
affected by yet-unknown complex physiological and regulatory variables (King et al., 2006). Competition between the alternate sigma factors RpoS and RpoD for binding to the RNA polymerase core has also been proposed to favour the appearance of mutations in rpoS (Farewell et al., 1998). These mutations affect the affinity of binding and are generally loss-of-function with little or no residual RpoS protein (Ferenci, 2008). No mutations were, however, found in $\operatorname{sig} B$ for any of the L. monocytogenes mutants analysed in our study. This suggests that selection pressure(s) may exist favouring loss-of-function mutations in elements of the SigB regulatory cascade rather than in the sigma factor itself.

Nucleotide polymorphisms have been associated with virulence defects in all $L$. monocytogenes lineages (Orsi et al., 2011). Indeed, while premature stop codons seem to be common in L. monocytogenes food isolates, these mutations are rare among human clinical isolates (Orsi et al., 2011). On the other hand, recent evidence shows that $L$. monocytogenes can adapt through selective mutations during in vitro and intra-host growth. Thus, L. monocytogenes possesses the ability to express growth advantage in the stationary phase through acquisition of mutations that optimize fitness during long-term stationary growth without negatively impacting virulence (Bruno \& Freitag, 2011). Regarding in vivo adaptation, intra-host environment(s) were reported to stimulate $r s b W$ mutations that impair SigB-dependent acid resistance together with invasion and growth of L. monocytogenes within macrophages and epithelial cells (Asakura et al., 2012). The identity of the selective pressures favouring these mutations is unknown. Recent studies have also provided genetic evidence for naturally occurring mutations in the $\operatorname{sig} B$ operon of $S$. aureus. Thus, natural S. aureus human isolates selected for high production of proteases and $\alpha$-haemolysin exhibit a SigB-deficient phenotype (Karlsson-Kanth et al., 2006). Nucleotide sequencing revealed that two of these strains had stop codons in $r s b U$ and $\operatorname{sig} B$ while the other strain had an insertion sequence element in $r s b U$. Adaptive evolution of $S$. aureus during chronic infection of a cystic fibrosis patient was also linked to a high mutation rate in loci associated with $\operatorname{sigB}$ (McAdam et al., 2011). As mentioned above, mutations in $\operatorname{sig} B$ seem, however, less favoured in L. monocytogenes. Our findings agree with recent microevolution analyses involving 195 L. monocytogenes isolates which revealed that $\operatorname{sig} B$ function is highly conserved (76 synonymous and 3 non-synonymous mutations) compared to actA (59 synonymous and 70 nonsynonymous mutations) or inlA (64 synonymous and 44 non-synonymous mutations) (den Bakker et al., 2008). Further studies should examine the ultimate basis of the apparent conservation of the sigB gene in L. monocytogenes, which seems not to be targeted by mutation at the same rate as in S. aureus. Notably, none of these studies has yet provided clues about the ultimate causative step(s) responsible for the emergence of these mutations in this important alternative sigma factor.
To our knowledge, this is the first report showing counterselection of SigB during mutant construction in $L$. monocytogenes. The similarity found between mutants in distinct LPXTG proteins ( $\Delta v i p, \Delta v i p:: \mathrm{km}$ and $\Delta i n l G)$ and some human isolates regarding the emergence of mutations in sigB-related genes suggests that common evolutionary pathways could be shared and that yet-unknown selective pressures could act on these loci resulting in a beneficial phenotype. Generation of deletion mutants is a common genetic procedure but, as demonstrated here for $L$. monocytogenes, it can inadvertently lead to inactivation of the SigB operon. A rapid chitinase-based test following the generation of any mutant may avoid subsequent work with strains carrying undesired mutations affecting SigB.

\section{ACKNOWLEDGEMENTS}

We thank Diana Barroso, Pablo García and Catarina Correia for their technical assistance. This work was supported by the following grants: from the Spanish Ministry of Economy and Competitiveness (Listress ERANet-Pathogenomics PIM2010EPA-00714; to F. G.P.); from the Spanish Ministry of Economy and Competitiveness (BIO2010-18962; to M.G.P.); from the Fundação para a Ciência e Tecnologia of Portugal, COMPETE and FEDER programmes (PTDC/SAU-MIC/ 111581/2009FCOMP-FEDER and ERANet-Pathogenomics Listress ERA-PTG/0003/2010; to D.C.); European Reseach Council advanced grant ERC-233348, Fondation Le Roch les Mousquetaires and Listress ERANet-Pathogenomics (ANR 2010-PATH-001-01) (to P.C.); and Listress ERANet-Pathogenomics Project from the German Federal Ministry of Education and Research (to T. C. ). L. B. M. and F. C. were supported by doctoral fellowships from FPU Program (Spanish Ministry of Education, Culture and Sports) and Fundação para a Ciência e Tecnologia of Portugal (SFRH/BD/61825/2009), respectively.

\section{REFERENCES}

Abram, F., Starr, E., Karatzas, K. A., Matlawska-Wasowska, K., Boyd, A., Wiedmann, M., Boor, K. J., Connally, D. \& O'Byrne, C. P. (2008a). Identification of components of the sigma $\mathrm{B}$ regulon in Listeria monocytogenes that contribute to acid and salt tolerance. Appl Environ Microbiol 74, 6848-6858.

Abram, F., Su, W. L., Wiedmann, M., Boor, K. J., Coote, P., Botting, C., Karatzas, K. A. \& O'Byrne, C. P. (2008b). Proteomic analyses of a Listeria monocytogenes mutant lacking sigmaB identify new components of the sigmaB regulon and highlight a role for sigmaB in the utilization of glycerol. Appl Environ Microbiol 74, 594-604.

Arnaud, M., Chastanet, A. \& Débarbouillé, M. (2004). New vector for efficient allelic replacement in naturally nontransformable, low-GCcontent, gram-positive bacteria. Appl Environ Microbiol 70, 68876891.

Asakura, H., Kawamoto, K., Okada, Y., Kasuga, F., Makino, S., Yamamoto, S. \& Igimi, S. (2012). Intrahost passage alters SigBdependent acid resistance and host cell-associated kinetics of Listeria monocytogenes. Infect Genet Evol 12, 94-101.

Atilano, M. L., Pereira, P. M., Yates, J., Reed, P., Veiga, H., Pinho, M. G. \& Filipe, S. R. (2010). Teichoic acids are temporal and spatial regulators of peptidoglycan cross-linking in Staphylococcus aureus. Proc Natl Acad Sci U S A 107, 18991-18996.

Begley, M., Sleator, R. D., Gahan, C. G. \& Hill, C. (2005). Contribution of three bile-associated loci, bsh, pva, and btlB, to gastrointestinal 
persistence and bile tolerance of Listeria monocytogenes. Infect Immun 73, 894-904.

Bierne, H. \& Cossart, P. (2007). Listeria monocytogenes surface proteins: from genome predictions to function. Microbiol Mol Biol Rev 71, 377-397.

Boneca, I. G., Dussurget, O., Cabanes, D., Nahori, M. A., Sousa, S., Lecuit, M., Psylinakis, E., Bouriotis, V., Hugot, J. P. \& other authors (2007). A critical role for peptidoglycan N-deacetylation in Listeria evasion from the host innate immune system. Proc Natl Acad Sci U S A 104, 997-1002.

Bruno, J. C., Jr \& Freitag, N. E. (2011). Listeria monocytogenes adapts to long-term stationary phase survival without compromising bacterial virulence. FEMS Microbiol Lett 323, 171-179.

Cabanes, D., Dussurget, O., Dehoux, P. \& Cossart, P. (2004). Auto, a surface associated autolysin of Listeria monocytogenes required for entry into eukaryotic cells and virulence. Mol Microbiol 51, 16011614.

Cabanes, D., Sousa, S., Cebriá, A., Lecuit, M., García-del Portillo, F. \& Cossart, P. (2005). Gp96 is a receptor for a novel Listeria monocytogenes virulence factor, Vip, a surface protein. EMBO J 24, 2827-2838.

Calvo, E., Pucciarelli, M. G., Bierne, H., Cossart, P., Albar, J. P. \& Garcia-Del Portillo, F. (2005). Analysis of the Listeria cell wall proteome by two-dimensional nanoliquid chromatography coupled to mass spectrometry. Proteomics 5, 433-443.

Chakraborty, T., Leimeister-Wächter, M., Domann, E., Hartl, M., Goebel, W., Nichterlein, T. \& Notermans, S. (1992). Coordinate regulation of virulence genes in Listeria monocytogenes requires the product of the prfA gene. J Bacteriol 174, 568-574.

Chaturongakul, S. \& Boor, K. J. (2006). SigmaB activation under environmental and energy stress conditions in Listeria monocytogenes. Appl Environ Microbiol 72, 5197-5203.

Cheung, A. L., Bayer, A. S., Zhang, G., Gresham, H. \& Xiong, Y. Q. (2004). Regulation of virulence determinants in vitro and in vivo in Staphylococcus aureus. FEMS Immunol Med Microbiol 40, 1-9.

Cossart, P. \& Toledo-Arana, A. (2008). Listeria monocytogenes, a unique model in infection biology: an overview. Microbes Infect 10, 1041-1050.

den Bakker, H. C., Didelot, X., Fortes, E. D., Nightingale, K. K. \& Wiedmann, M. (2008). Lineage specific recombination rates and microevolution in Listeria monocytogenes. BMC Evol Biol 8, 277.

Doumith, M., Cazalet, C., Simoes, N., Frangeul, L., Jacquet, C., Kunst, F., Martin, P., Cossart, P., Glaser, P. \& Buchrieser, C. (2004). New aspects regarding evolution and virulence of Listeria monocytogenes revealed by comparative genomics and DNA arrays. Infect Immun 72, 1072-1083.

Dramsi, S., Biswas, I., Maguin, E., Braun, L., Mastroeni, P. \& Cossart, P. (1995). Entry of Listeria monocytogenes into hepatocytes requires expression of inIB, a surface protein of the internalin multigene family. Mol Microbiol 16, 251-261.

Dussurget, O., Cabanes, D., Dehoux, P., Lecuit, M., Buchrieser, C., Glaser, P., Cossart, P. \& European Listeria Genome Consortium (2002). Listeria monocytogenes bile salt hydrolase is a PrfA-regulated virulence factor involved in the intestinal and hepatic phases of listeriosis. Mol Microbiol 45, 1095-1106.

Farewell, A., Kvint, K. \& Nyström, T. (1998). Negative regulation by RpoS: a case of sigma factor competition. Mol Microbiol 29, 10391051.

Ferenci, T. (2008). The spread of a beneficial mutation in experimental bacterial populations: the influence of the environment and genotype on the fixation of rpoS mutations. Heredity (Edinb) 100, $446-452$.
Garcia-del Portillo, F., Calvo, E., D’Orazio, V. \& Pucciarelli, M. G. (2011). Association of ActA to peptidoglycan revealed by cell wall proteomics of intracellular Listeria monocytogenes. J Biol Chem 286, 34675-34689.

Giotis, E. S., Julotok, M., Wilkinson, B. J., Blair, I. S. \& McDowell, D. A. (2008). Role of sigma B factor in the alkaline tolerance response of Listeria monocytogenes $10403 \mathrm{~S}$ and cross-protection against subsequent ethanol and osmotic stress. J Food Prot 71, 1481-1485.

Glaser, P., Frangeul, L., Buchrieser, C., Rusniok, C., Amend, A., Baquero, F., Berche, P., Bloecker, H., Brandt, P. \& other authors (2001). Comparative genomics of Listeria species. Science 294, 849852.

Guzman, C. A., Rohde, M., Chakraborty, T., Domann, E., Hudel, M., Wehland, J. \& Timmis, K. N. (1995). Interaction of Listeria monocytogenes with mouse dendritic cells. Infect Immun 63, 3665-3673.

Hain, T., Chatterjee, S. S., Ghai, R., Kuenne, C. T., Billion, A., Steinweg, C., Domann, E., Kärst, U., Jänsch, L. \& other authors (2007). Pathogenomics of Listeria spp. Int J Med Microbiol 297, 541-557.

Hain, T., Hossain, H., Chatterjee, S. S., Machata, S., Volk, U., Wagner, S., Brors, B., Haas, S., Kuenne, C. T. \& other authors (2008). Temporal transcriptomic analysis of the Listeria monocytogenes EGDe sigmaB regulon. BMC Microbiol 8, 20.

Hamon, M., Bierne, H. \& Cossart, P. (2006). Listeria monocytogenes: a multifaceted model. Nat Rev Microbiol 4, 423-434.

Hanson, B. R. \& Neely, M. N. (2012). Coordinate regulation of Grampositive cell surface components. Curr Opin Microbiol 15, 204-210.

Hecker, M., Pané-Farré, J. \& Völker, U. (2007). SigB-dependent general stress response in Bacillus subtilis and related gram-positive bacteria. Annu Rev Microbiol 61, 215-236.

Karlsson-Kanth, A., Tegmark-Wisell, K., Arvidson, S. \& Oscarsson, J. (2006). Natural human isolates of Staphylococcus aureus selected for high production of proteases and alpha-hemolysin are sigmaB deficient. Int J Med Microbiol 296, 229-236.

Kazmierczak, M. J., Wiedmann, M. \& Boor, K. J. (2006). Contributions of Listeria monocytogenes sigmaB and PrfA to expression of virulence and stress response genes during extra- and intracellular growth. Microbiology 152, 1827-1838.

King, T., Seeto, S. \& Ferenci, T. (2006). Genotype-by-environment interactions influencing the emergence of rpos mutations in Escherichia coli populations. Genetics 172, 2071-2079.

Kirchner, M. \& Higgins, D. E. (2008). Inhibition of ROCK activity allows InlF-mediated invasion and increased virulence of Listeria monocytogenes. Mol Microbiol 68, 749-767.

Larsen, M. H., Leisner, J. J. \& Ingmer, H. (2010). The chitinolytic activity of Listeria monocytogenes EGD is regulated by carbohydrates but also by the virulence regulator PrfA. Appl Environ Microbiol 76, 6470-6476.

Lingnau, A., Domann, E., Hudel, M., Bock, M., Nichterlein, T., Wehland, J. \& Chakraborty, T. (1995). Expression of the Listeria monocytogenes EGD inlA and inlB genes, whose products mediate bacterial entry into tissue culture cell lines, by PrfA-dependent and -independent mechanisms. Infect Immun 63, 3896-3903.

Loh, E., Gripenland, J. \& Johansson, J. (2006). Control of Listeria monocytogenes virulence by $5^{\prime}$-untranslated RNA. Trends Microbiol 14, 294-298.

Mariscotti, J. F., Quereda, J. J. \& Pucciarelli, M. G. (2012). Contribution of sortase A to the regulation of Listeria monocytogenes LPXTG surface proteins. Int Microbiol 15, 43-51.

Marraffini, L. A., Dedent, A. C. \& Schneewind, O. (2006). Sortases and the art of anchoring proteins to the envelopes of gram-positive bacteria. Microbiol Mol Biol Rev 70, 192-221. 
McAdam, P. R., Holmes, A., Templeton, K. E. \& Fitzgerald, J. R. (2011). Adaptive evolution of Staphylococcus aureus during chronic endobronchial infection of a cystic fibrosis patient. PLOS ONE 6, e24301.

Mengaud, J., Lecuit, M., Lebrun, M., Nato, F., Mazie, J. C. \& Cossart, P. (1996). Antibodies to the leucine-rich repeat region of internalin block entry of Listeria monocytogenes into cells expressing E-cadherin. Infect Immun 64, 5430-5433.

Novick, R. P. (2003). Autoinduction and signal transduction in the regulation of staphylococcal virulence. Mol Microbiol 48, 1429-1449.

O'Byrne, C. P. \& Karatzas, K. A. (2008). The role of sigma B (sigma B) in the stress adaptations of Listeria monocytogenes: overlaps between stress adaptation and virulence. Adv Appl Microbiol 65, 115-140.

Orsi, R. H., den Bakker, H. C. \& Wiedmann, M. (2011). Listeria monocytogenes lineages: genomics, evolution, ecology, and phenotypic characteristics. Int J Med Microbiol 301, 79-96.

Palmer, M. E., Wiedmann, M. \& Boor, K. J. (2009). Sigma(B) and sigma(L) contribute to Listeria monocytogenes $10403 \mathrm{~S}$ response to the antimicrobial peptides SdpC and nisin. Foodborne Pathog Dis 6, 1057-1065.

Personnic, N., Bruck, S., Nahori, M. A., Toledo-Arana, A., Nikitas, G., Lecuit, M., Dussurget, O., Cossart, P. \& Bierne, H. (2010). The stressinduced virulence protein InlH controls interleukin-6 production during murine listeriosis. Infect Immun 78, 1979-1989.

Price C. W. (2010). General stress response in Bacillus subtilis and related Gram-positive bacteria, p. 301-318. In Storz G., Hengge R. (ed.), Bacterial stress responses, 2nd ed. ASM Press, Washington, DC.

Pucciarelli, M. G., Calvo, E., Sabet, C., Bierne, H., Cossart, P. \& Garcia-del Portillo, F. (2005). Identification of substrates of the Listeria monocytogenes sortases A and B by a non-gel proteomic analysis. Proteomics 5, 4808-4817.

Raengpradub, S., Wiedmann, M. \& Boor, K. J. (2008). Comparative analysis of the sigma B-dependent stress responses in Listeria monocytogenes and Listeria innocua strains exposed to selected stress conditions. Appl Environ Microbiol 74, 158-171.

Raffelsbauer, D., Bubert, A., Engelbrecht, F., Scheinpflug, J., Simm, A., Hess, J., Kaufmann, S. H. \& Goebel, W. (1998). The gene cluster
inlC2DE of Listeria monocytogenes contains additional new internalin genes and is important for virulence in mice. Mol Gen Genet 260, 144-158.

Reis, O., Sousa, S., Camejo, A., Villiers, V., Gouin, E., Cossart, P. \& Cabanes, D. (2010). LapB, a novel Listeria monocytogenes LPXTG surface adhesin, required for entry into eukaryotic cells and virulence. $J$ Infect Dis 202, 551-562.

Sabet, C., Lecuit, M., Cabanes, D., Cossart, P. \& Bierne, H. (2005). LPXTG protein InlJ, a newly identified internalin involved in Listeria monocytogenes virulence. Infect Immun 73, 6912-6922.

Schlag, M., Biswas, R., Krismer, B., Kohler, T., Zoll, S., Yu, W., Schwarz, H., Peschel, A. \& Götz, F. (2010). Role of staphylococcal wall teichoic acid in targeting the major autolysin Atl. Mol Microbiol 75, 864-873.

Spirig, T., Weiner, E. M. \& Clubb, R. T. (2011). Sortase enzymes in Gram-positive bacteria. Mol Microbiol 82, 1044-1059.

Swaminathan, B. \& Gerner-Smidt, P. (2007). The epidemiology of human listeriosis. Microbes Infect 9, 1236-1243.

Toledo-Arana, A., Dussurget, O., Nikitas, G., Sesto, N., GuetRevillet, H., Balestrino, D., Loh, E., Gripenland, J., Tiensuu, T. \& other authors (2009). The Listeria transcriptional landscape from saprophytism to virulence. Nature 459, 950-956.

Vázquez-Boland, J. A., Kuhn, M., Berche, P., Chakraborty, T., Dominguez-Bernal, G., Goebel, W., González-Zorn, B., Wehland, J. \& Kreft, J. (2001). Listeria pathogenesis and molecular virulence determinants. Clin Microbiol Rev 14, 584-640.

Wilson, K. (2001). Preparation of genomic DNA from bacteria. In Current Protocols in Molecular Biology, pp. 2.4.1.-2.4.5. Edited by F. M. Ausubel, R. Brent, R. E. Kingston, D. D. Moore, J. G. Seidman, J. A. Smith \& K. Struhl. New York: John Wiley \& Sons.

Wouters, J. A., Hain, T., Darji, A., Hüfner, E., Wemekamp-Kamphuis, H., Chakraborty, T. \& Abee, T. (2005). Identification and characterization of di- and tripeptide transporter DtpT of Listeria monocytogenes EGD-e. Appl Environ Microbiol 71, 5771-5778.

Edited by: H. Ingmer 